\section{Bilateral Pallidotomy by Using Deep Brain Stimulation Electrode in Parkinson's Disease}

\section{Abstract}

Deep brain stimulation (DBS) is a safe and effective treatment in alleviating motor symptoms and complications in advanced Parkinson's disease (PD). However, in some patients, the DBS system may need to be removed due to recurrent hardware infection. Here we report a case of a patient who had frequent internal pulse generator (IPG) site infections and eventually underwent bilaterally pallidotomy using previously implanted globus pallidus interna (GPi) DBS electrodes. A 57-yearold woman with advanced PD and was implanted bilateral subthalamic nucleus (STN) DBS. 18 months after she suffered from new onset loss of postural reflexes and frequent falls that were directly related with STN stimulation. Therefore, the STN electrodes were swithched to GPiand one year after switching to GPi DBS, she developed severe infection and skin erosion over the DBS hardware. The hardware had to be removed due to recurrent infections and before explanting the electrodes, bilateral pallidotomy with radiofrequency was performed by using the existing DBS electrodes. Two years after the bilateral posteroventral pallidotomy, the patient had good and stable control of PD. To our knowledge, this is the first case report where existing DBS electrodes were used for bilateral pallidotomy by RF. This technique is safe and efficient, but further studies and large series are needed to confirm its long-term benefits.

Keywords: Deep brain stimulation; Parkinson's disease; Radiofrequency lesion; Pallidotomy

\section{Göksemin Acar ${ }^{1}$, Selçuk Göçmen², Murat Kocaoğlu' ${ }^{1}$, Serkan Civlan' ${ }^{1}$ Athanasios Zisakis ${ }^{1}$ and Feridun Acar ${ }^{1}$

\author{
1 Pamukkale University, Denizli, Turkey \\ 2 Denizli Surgery Hospital, Denizli, \\ Turkey
}

\section{Corresponding author: Göksemin Acar}

इ goksemind@yahoo.com

Associate Professor of Neurology, Department of Neurology, Pamukkale University, Kınıklı Campus, Denizli, Turkey.

Tel: +90258266 60 00- 5682

Citation: Acar G, Göçmen S, Kocaoğlu M, et al. Bilateral Pallidotomy by Using Deep Brain Stimulation Electrode in Parkinson's Disease. Neurosurg. 2016, 1:2.

Received: April 02, 2016; Accepted: May 18, 2016; Published: May 25, 2016

\section{Introduction}

Stereotactic lesioning of the thalamus and basal ganglia for treatment movement disorders is a well-known procedure that was frequently applied prior to the introduction of deep brain stimulation (DBS). However it is irreversible and there is a higher incidence of side effects, therefore DBS has outbalanced with its efficiency and safety as the first line surgical treatment in movement disorders [1, 2].

Although rare, in case of DBS hardware-related complications, hardware should be removed and usually after a period followed by either repeated DBS implantation or a lesioning procedure [1-3]. Recently, studies have focused on radiofrequency (RF) lesions by using DBS electrodes in case of hardware erosion, infection, or lack of efficacy before removing the system [1-4]. The cases, which have been reported so far, were unilateral RF lesioning by using a previously implanted DBS electrode. Here we report a case of advanced Parkinson's disease (PD) with bilateral pallidotomy generated by RF using previously implanted globus pallidus interna (GPi) DBS electrodes.

\section{Patient and Methods}

A 57-year-old woman who was diagnosed as PD since 20 years and has been put on dopaminergic drugs. Six years after the first symptoms she developed gait disturbances and motor complications. After 9 years of symptoms' onset she had her first DBS implanted to bilateral subthalamic nucleus (STN) (Figure 1). She responded excellent to STN-DBS and her motor fluctuations were minimal, disabling peak dose dyskinesias have subsided and her medication "on" DBS "on" UPDRS was 10. However, after 2 months she came back with high fever and skin erosion at the internal pulse generator (IPG) site and the system had to be removed and reimplanted 5 months later. Eighteen months after reimplantation she suffered from a new onset postural instability and described frequent falls. She had no postural reflex and posturography revealed $100 \%$ falling risk at medication "on" DBS 
"on" state. At DBS "off" (after $36 \mathrm{~h}$ of washout) and medication "on" state, she had a better postural response and posturography revealed $80 \%$ falling risk Her postural instability and frequent falls might be due to either disease progression or direct effect of STN stimulation. At this point we decided that her new symptoms were related with STN stimulation and switched the electrodes to globus pallidus interna (GPi). After GPi DBS not only her DBS "on" medication "on" state motor scores were similar to that of STN DBS (12 vs. 10) but also she instantly regained her postural reflex. Posturography revealed that falling risk was reduced to $60 \%$. Unfortunately, one year after GPi DBS operation she had a severe infection at IPG site. Therefore we discussed the removal of the system permanently and to make bilateral pallidotomy via existing DBS electrodes (3387-40; Medtronic, Inc., Minneapolis, Minn., USA) by using radiofrequency.

IPG was shut off 2 days before the operation for washout. Under local anesthesia, after externalizing the DBS electrodes, they were connected to the radiofrequency (RF) generator (NT-1100; Neurotherm Medical Inc., Boston, USA). The RF generator was used for bilateral test stimulation and according to the patient's most recent settings, right hand tremor arrested at $4 \mathrm{~V}$ and left hand tremor arrested at $3 \mathrm{~V}$ without additional associated neurological symptoms. On the basis of clinical response, we created bipolar lesions bilaterally (at $100 \mathrm{~mA}$ for $60 \mathrm{~s}$ ) and then the tip was withdrawn $2 \mathrm{~mm}$ and heated for another $60 \mathrm{~s},(620$ impedence, $100 \mathrm{~mA}, 60 \mathrm{~s}, 150 \mathrm{~Hz}, 0.2$ stimulation, 0-3 V). Lesion positions and sizes were confirmed with magnetic resonance imaging which showed perilesional edema in a small degree (Figure 2).

At the end of surgery the electrodes and IPG were removed, and the early postoperative CT excluded GPi complications including bleeding. After bilateral pallidotomy, the patient had good and stable control of PD at $3^{\text {rd }}, 6^{\text {th }}$ and $18^{\text {th }}$ months. DBS "on" medication "on" state UPDRS motor scores were 22, 23 and 25, respectively. She presented no motor or cognitive complications after the operation.

\section{Discussion}

The clinical benefit provided by DBS is significant; however, hardware infections may lead to removal of the DBS $[5,6]$. In these cases lesioning surgery can be an option. Unilateral pallidotomy has been shown to be safe and effective treatment for the contralateral motor symptoms of PD [2]. However, many patients with PD suffer from bilateral disabling motor symptoms. Some preliminary studies reported that bilateral pallidotomy improved dyskinesia and decreased "off" time in PD patients, but they also noted that the rate of speech and cognitive complications were higher after bilateral pallidotomy [7-9]. Several studies have reported that bilateral pallidotomy resulted with unacceptable adverse effects [8-10]. During these procedures, major adverse effects were associated with the technique used for stereotactic surgery and the side effects, such as speech disturbance and memory dysfunction were related with bilateral lesions, suggesting these complications outweigh the benefits from surgery. On the other hand, others have reported that bilateral

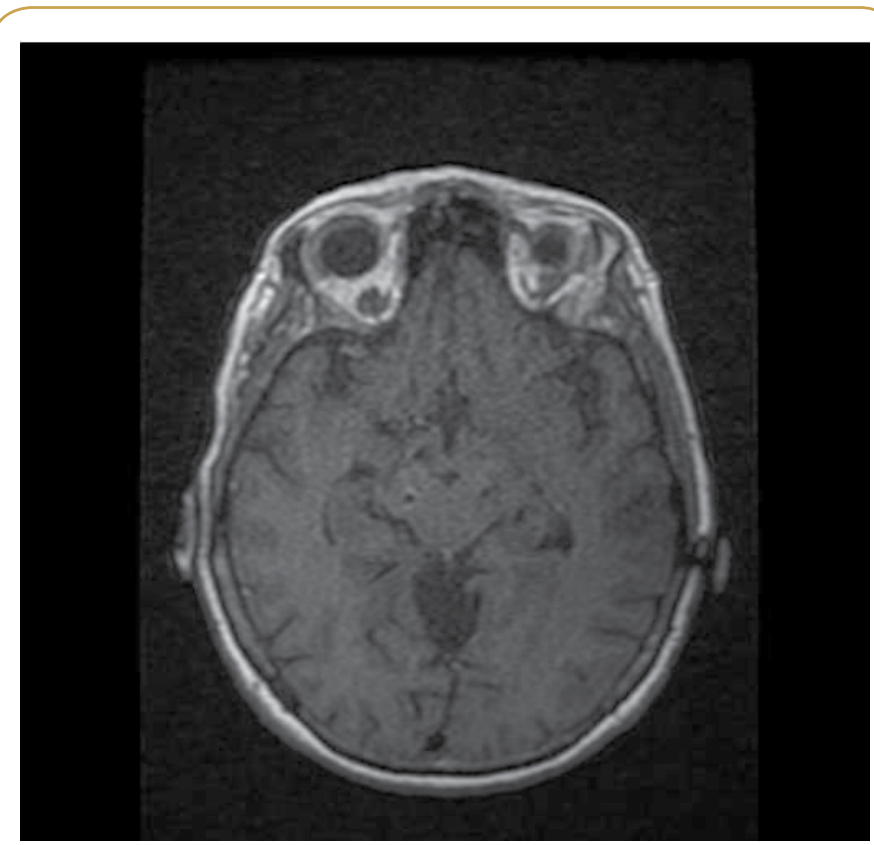

Figure 1 T1 weighted axial MRI showing the DBS electrodes in bilateral STN.

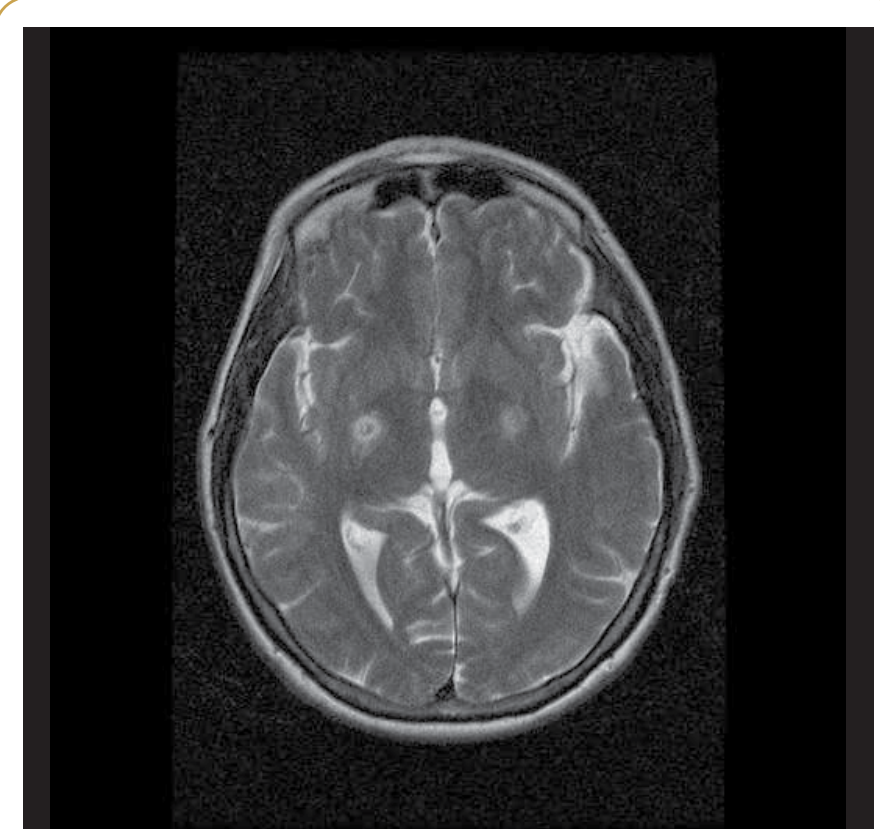

Figure 2 T2 weighted axial MRI showing the bilateral lesions in GPi created by RF via existing GPi BS electrodes.

pallidotomy was safe and effective as unilateral procedure $[7,11-$ 14]. In our case, bilateral pallidotomy was carried out via existing DBS electrodes that are effectively alleviating the parkinsonian symptoms. During the surgery, macrostimulation was carried out to assure the safe contacts for lesioning. Recently, Franzini et al. performed local field potential (LFP) recording through implanted DBS electrode before the staged bilateral pallidotomy with RF. They emphasized that LFP recording was an objective proof of the lesion at the target before clinical evidence and selectivity of the lesion [15]. 
In conclusion, a lesioning procedure can always be considered if DBS fails. In cases in which DBS has to be removed due to hardware erosion, infection, or lack of efficacy, RF lesioning procedures by using DBS electrodes might be performed unilaterally or bilaterally before removing the system. Furthermore, DBS electrodes can also be used for LFP recordings to confirm the lesion selectivity and localization before lesioning. We believe that this technique appears to be safe and efficient and can be a reasonable alternative in certain situations, but further studies and large series are needed to confirm its long-term benefits in advanced PD patients. 


\section{References}

1 Bulluss KJ, Pereira EA, Joint C, Aziz TZ (2013) Pallidotomy after chronic deep brain stimulation. Neurosurg Focus 35: E5.

2 Oh MY, Abosch A, Kim SH, Lang AE, Lozano AM (2002) Longterm hardware-related complications of deep brain stimulation. Neurosurgery 50: 1268-1274.

3 Marras C, Zorzi G, Lenardi C, Rizzi M, Messina G, et al. (2009) Deep brain stimulation electrode used for radiofrequency lesion of the globus pallidus internus in dystonia. Stereotact Funct Neurosurg 87: 348-352.

4 Strickland BA, Jimenez-Shahed J, Jankovic J, Viswanathan A (2013) Radiofrequency lesioning through deep brain stimulation electrodes: a pilot study of lesion geometry and temperature characteristics. J Clin Neurosci 20: 1709-1712.

5 Temel Y, Ackermans L, Celik H, Spincemaille GH, van der Linden C, et al. (2004) Management of hardware infections following deep brain stimulation. Acta Neurochir (Wien) 146: 355-361.

6 Voges J, Waerzeggers Y, Maarouf M, Lehrke R, Koulousakis A, et al. (2006) Deep-brain stimulation: long-term analysis of complications caused by hardware and surgery--experiences from a single centre. J Neurol Neurosurg Psychiatry 77: 868-872.

7 Counihan TJ, Shinobu LA, Eskandar EN, Cosgrove GR, Penney JB Jr (2001) Outcomes following staged bilateral pallidotomy in advanced Parkinson's disease. Neurology 56: 799-802.

8 Intemann PM, Masterman D, Subramanian I, DeSalles A, Behnke E, et al. (2001) Staged bilateral pallidotomy for treatment of Parkinson disease. J Neurosurg 94: 437-444.

9 Scott R, Gregory R, Hines N, Carroll C, Hyman N, et al. (1998) Neuropsychological, neurological and functional outcome following pallidotomy for Parkinson's disease. A consecutive series of eight simultaneous bilateral and twelve unilateral procedures. Brain 121: 659-675.

10 Svennilson E, Torvik A, Lowe R, Leksell L (1960) Treatment of parkinsonism by stereotatic thermolesions in the pallidal region. A clinical evaluation of 81 cases. Acta Psychiatr Scand 35: 358-377.

11 Chung SJ, Hong SH, Kim SR, Lee MC, Jeon SR (2006) Efficacy and safety of simultaneous bilateral pallidotomy in advanced Parkinson's disease. Eur Neurol 56: 113-118.

12 Favre J, Burchiel KJ, Taha JM, Hammerstad J (2000) Outcome of unilateral and bilateral pallidotomy for Parkinson's disease: patient assessment. Neurosurgery 46: 344-353.

13 lacono RP, Lonser RR, Kuniyoshi S (1995) Unilateral versus bilateral simultaneous posteroventral pallidotomy in subgroups of patients with Parkinson's disease. Stereotact Funct Neurosurg 65: 6-9.

14 Schuurman PR, de Bie RM, Speelman JD, Bosch DA (1997) Bilateral posteroventral pallidotomy in advanced Parkinson's disease in three patients. Mov Disord 12: 752-755.

15 Franzini A, Cordella R, Penner F, Rosa M, Messina G, et al. (2015) Posteroventrolateral pallidotomy through implanted DBS electrodes monitored by recording local field potentials. Br J Neurosurg 17: 1-3. 